\title{
A current affair in MS
}

\section{By Tracey Baas, Senior Editor}

A European team has used the diabetes drug glibenclamide to block inflammation-induced neurodegeneration in mice. ${ }^{1}$ The team plans to test the nonspecific inhibitor of the cationic channel TRPM4 in an investigator-led clinical trial in multiple sclerosis and is looking for pharmas to develop more specific antagonists of the target.

Transient receptor potential cation channel subfamily $\mathrm{M}$ member 4 (TRPM4) is a widely expressed $\mathrm{Ca}^{2+}$-activated, voltage-dependent cation channel that depolarizes the plasma membrane by increasing sodium influx.

A group of German and Swiss researchers hypothesized that TRPM4 might be a key player in MS because disease-associated inflammation leads, in part,

"I think the work opens new perspectives on the role of the ion channel in MS pathology, and I believe in the efficacy of glibenclamide. The team will have to be diligent in monitoring possible physiopathology in multiple organs since this channel has a broad regulatory effect."

-Pierre Launay, Institut National de la Santé et de la Recherche Médicale to elevated glutamate levels, which promote excitotoxic neurodegeneration by inducing $\mathrm{Na}^{+}$and $\mathrm{Ca}^{2+}$ influx. ${ }^{2}$

Indeed, the ion channels NaV1.6 ( PN4; SCN8A), $\mathrm{Na}^{+}-\mathrm{Ca}^{2+}$ exchanger and acidsensing ion channel-1 (ASIC1) are dysregulated in $\mathrm{MS},{ }^{3-5}$ but nothing was known about the role TRPM4 might play in the disease.

In Trpm4 knockout mice with experimental autoimmune encephalomyelitis (EAE), compared with wild-type EAE mice, the team saw an overall decrease in disease severitydespite similar immune cell activation and infiltration.

Brain samples from both EAE wild-type mice and patients with MS showed that TRPM4 expression was greater within active demyelinating brain lesions than inactive lesions or tissue adjacent to active lesions.

The unanswered question was if-and how-Trpm 4 affected the way in which neurons responded to toxic insults.

In assays to monitor ion channel activity, wild-type murine hippocampal neurons showed higher current density and electrical capacitance than Trpm4 knockout hippocampal neurons after receiving neurotoxic levels of glutamate. The lower levels of flowing current indicate that the $\operatorname{Trpm} 4^{-/-}$neurons are protected from the induction of excitotoxicity.
Further analysis of the wild-type neurons showed that the increase in electrical capacitance corresponded to swelling of the cell and a loss of cell integrity. Conversely, the Trpm 4 knockout neurons showed similar cell volumes and integrity in glutamate-treated and untreated conditions.

Axonal swellings occur in the brains of patients with $\mathrm{MS}^{6}$ and in the spinal cords of mice with EAE, ${ }^{7,8}$ suggesting TRPM4 may contribute to axonal injury and pathogenesis of MS.

To test the effects of pharmacological inhibition of the target, the team turned to glibenclamide, a generic sulfonylurea that blocks multiple ion channels, including TRPM4. ${ }^{9-11}$

In wild-type EAE mice, glibenclamide decreased axonal and neuronal degeneration and clinical disease scores compared with vehicle without affecting CNS immune responses. In vitro testing of wild-type mouse neurons showed that glibenclamide also inhibited glutamate-mediated cell swelling and excitotoxicity.

Results were published in Nature Medicine.

The team included researchers from the University Medical Center Hamburg-Eppendorf, Geneva University Hospitals, the University of Geneva, Catholic University Leuven, Saarland University, the University of Goettingen and Heidelberg University.

"No one had been paying attention to TRPM4 in MS, and targeting TRPM4 provides therapeutic benefit in a manner separate from the classical immune intervention that many groups are going after," said Lawrence Steinman, professor of neurology and neurological sciences, pediatrics and genetics at Stanford University School of Medicine.

"I would like to see what happens when ion channel blockers to suppress axon and neuronal damage caused by glutamate-induced neurotoxicity are used in conjunction with immunomodulators," added Steinman.

"I think focusing on a single drug with pleotropic effects is the best strategy" in MS, said Marc Simard, professor of neurosurgery, pathology and physiology at the University of Maryland School of Medicine. "Glibenclamide is such a drug." Simard added that not only does glibenclamide block TRPM4 but also there is good evidence that it exerts anti-inflammatory effects, reducing neutrophil infiltration.

"I think the work opens new perspectives on the role of the ion channel in MS pathology, and I believe in the efficacy of glibenclamide," added Pierre Launay, who pioneered the use of glibenclamide to target TRPM4 and is a research director at the Institut National de la Santé et de la Recherche Médicale (INSERM). "The team will have to be diligent in monitoring possible physiopathology in multiple organs since this channel has a broad regulatory effect."

\section{Into the clinic}

"We want to start an investigator-led trial of glibenclamide in MS but will need to secure financing first," said Manuel Friese, leader of the 
German/Swiss team and group leader of an Emmy Noether Research Group at the Center for Molecular Neurobiology Hamburg at the University Medical Center Hamburg-Eppendorf.

Glibenclamide is the second drug the group has repurposed for MS. In 2011, the team showed that the hypertension drug amiloride blocked ASIC1 and staved off inflammation-induced neurodegeneration. ${ }^{4}$

An investigator-led trial in patients with primary-progressive MS (PPMS) has further shown that amiloride may exert neuroprotective effects. The findings will be published in the near future in Brain.

"In PPMS patients, we observed a significant decrease in normalized annual rate of whole-brain volume during the treatment phase compared to the pretreatment phase," said Lars Fugger, professor of clinical neurosciences at The Weatherall Institute of Molecular Medicine at University of Oxford. "Consistent with this decrease, we showed that changes in diffusion indices of tissue damage within major, clinically relevant white matter and deep grey matter structures was also significantly decreased during the treatment phase."

The researchers now are enrolling patients with MS who have optic neuritis in a Phase IIb trial of amiloride. The study is organized and coordinated by Fugger and Matt Craner, clinical lead of the MS Clinical Trials Unit at the University of Oxford.

The German/Swiss team also is exploring the use of glibenclamide in other animal models of disease in which glutamate excitotoxicity is important for pathogenesis, such as Parkinson's disease (PD), Alzheimer's disease (AD), amyotrophic lateral sclerosis (ALS) and stroke. Friese declined to give further details.

A patent application has been filed by University Medical Center Hamburg-Eppendorf for the use of TRPM4 inhibitors to treat MS. The work is not licensed, but the university is looking for pharmaceutical companies to develop specific inhibitors for TRPM4.
Baas, T. SciBX 5(47); doi:10.1038/scibx.2012.1223

Published online Dec. 6, 2012

\section{REFERENCES}

1. Schattling, B. et al. Nat. Med.; published online Nov. 18, 2012; doi:10.1038/nm.3015

Contact: Manuel A. Friese, University Medical Center HamburgEppendorf, Hamburg, Germany e-mail: manuel.friese@zmnh.uni-hamburg.de

2. Lau, A. \& Tymianski, M. Pflugers Arch. 460, 525-542 (2010)

3. Craner, M.J. et al. Proc. Natl. Acad. Sci. USA 101, 8168-8173 (2004)

4. Friese, M.A. et al. Nat. Med. 13, 1483-1489 (2007)

5. Vergo, S. et al. Brain 134, 571-584 (2011)

6. Fisher, E. et al. Ann. Neurol. 62, 219-228 (2007)

7. Nikić, l. et al. Nat. Med. 17, 495-499 (2011)

8. Stirling, D.P. \& Stys, P.K. Trends Mol. Med. 16, 160-170 (2010)

9. Demion, M. et al. Cardiovasc. Res. 73, 531-538 (2007)

10. Becerra, A. et al. Cardiovasc. Res. 91, 677-684 (2011)

11. Chen, M. et al. J. Neurosci. 23, 8568-8577 (2003)

COMPANIES AND INSTITUTIONS MENTIONED

Catholic University Leuven, Leuven, Belgium

Geneva University Hospitals, Geneva, Switzerland

Heidelberg University, Heidelberg, Germany Institut National de la Santé et de la Recherche Médicale, Paris, France

Saarland University, Homburg, Germany

Stanford University School of Medicine, Stanford, Calif. University Medical Center Hamburg-Eppendorf, Hamburg, Germany

University of Geneva, Geneva, Switzerland

University of Goettingen, Goettingen, Germany

University of Maryland School of Medicine, Baltimore, Md.

University of Oxford, Oxford, U.K.

The Weatherall Institute of Molecular Medicine at University of Oxford, Oxford, U.K. 